\title{
Targeted therapy for lung cancer
}

\author{
Anne-Pascale Meert ${ }^{1}$ and Jacques Cadranel $^{2}$
}

Affiliations: 'Service des Soins Intensifs et Urgences Oncologiques \& Oncologie Thoracique, Institut Jules Bordet, Université Libre de Bruxelles (ULB), Brussels, Belgium. ${ }^{2}$ Service de Pneumologie et Centre Expert en Oncologie Thoracique, AP-HP Hôpital Tenon and P\&M Paris 6 University, Paris, France.

Correspondence: A-P. Meert, 1 rue Héger Bordet, Institut Jules Bordet, B-1000 Brussels, Belgium. E-mail: ap.meertabordet.be

$@$ ERSpublications

Targeted therapy for lung cancer: ERS promotes exchange of knowledge http://ow.ly/RV285

Thoracic malignancies are a menacing worldwide health problem. To treat lung cancer optimally, care must be prompt, multidisciplinary, and patient-centred. In the entire process, pulmonologists have a key role. Pulmonologists and their professional societies should also enhance lung cancer research and education to provide better treatment options and patient care [1].

To meet that challenge, an action plan [2] was designed by the European Respiratory Society (ERS) Thoracic Oncology Assembly officers in March 2010, which was subsequently approved and strongly welcomed by the ERS Executive Committee. Today the Thoracic Oncology Assembly has almost achieved all of the following. 1) To create a Task Force for a European initiative for quality management in lung cancer care [3]. 2) To develop a specific training in thoracic oncology [4]. The HERMES (Harmonised Education in Respiratory Medicine for European Specialists) syllabus for thoracic oncology has been published and the curriculum will be finalised this year. 3) To increase the number of meaningful publications in the ERS journals. 4) To develop relationships with other European societies, such as the European Society for Medical Oncology, the European Society for Therapeutic Radiology and Oncology, the European Society of Thoracic Surgery, the European society of Radiology, the European Association for Cardio-Thoracic Surgery. 5) To promote research in thoracic oncology. With this in mind, the Thoracic Oncology Assembly leaders decided to build an ERS Research Seminar on Targeted Therapy for Lung Cancer.

The ERS Research Seminar took place in Paris (France) May 6-7, 2015. The main aim of this seminar was to promote exchange of knowledge and interaction between basic scientists and clinicians as well as between the different research groups that are currently working on targeted therapy for lung cancer. The participants had, throughout the seminar, the opportunities to evaluate advances in lung cancer targeted therapy, to discuss controversial issues, and to define the topics of future research projects aiming to improve the efficacy of targeted drugs as well as access to larger subgroups of patients. Consequently, a large amount of time was dedicated to discussion. We hosted 85 participants from all around the world: Europe (15 countries), Asia (two countries), North America (two countries) and South America (one country) and Africa (two countries).

The first session of the seminar was dedicated to molecular biology of lung cancer. Philippe Schnabel (Instit. Allgemeine \& Spezielle Pathologie Universitätsklinikum des Saarlandes, Hamburg, Germany) reviewed the most frequent molecular abnormalities linked to the different histopathology in lung cancer and Fernando Gamarra (MVZ Klinikum Straubing GmbH, Pneumologie, Munich, Germany) reviewed the latest techniques to extract the tissue (radial endobronchial ultrasound (EBUS), electromagnetic navigation, and cryobiopsies). Rafael Rosell (Catalan Institute of Oncology Hospital Germans Trias i Pujol, Barcelona, Spain) gave a brilliant talk on the possible use of liquid biopsy to assess epidermal growth factor receptor (EGFR) mutations on circulating free (cf) DNA and to correlate EGFR mutation cfDNA with outcome.

Received: May 222015 | Accepted after revision: June 152015

Conflict of interest: Disclosures can be found alongside the online version of this article at erj.ersjournals.com

Copyright @ERS 2015 
The second session was dedicated to EGFR mutations. Nicolas Girard (Sce de pneumologie du Pr Cordier, Hopital Louis Pradel U80, Lyon, France) provided an extended review of all tyrosine kinase transmembrane alterations in nonsmall cell lung cancer (NSCLC) (EGFR, anaplastic lymphoma kinase (ALK), human epidermal growth factor receptor (HER) 2, ROS1, mesenchymal-epithelial transition (MET), rearranged during transfection (RET), fibroblast growth factor receptor (FGFR) 1, discovering domain receptor 2). Jacques Cadranel showed us that there is a discrepancy between the recommendations for EGFR mutation testing and EGFR tyrosine kinase inhibitors (TKI) using and the practices in real life. Some countries have limited access to EGFR testing and in countries where the test is available it is only performed in $20-60 \%$ of the patients. Moreover, he raised the question concerning the reason for non-treatment of the $10 \%$ of patients with the EGFR mutation. He also presented recent data that suggested a differential efficacy of TKI depending on the type of EGFR mutation (del19 versus L858R point mutation) leading to thought on optimising therapeutic sequences in this population. Rafael Rossell reviewed the different mechanisms of EGFR TKI failure (T790M gatekeeper mutation, activation of alternative TKI receptor, which maintains signalling of key downstream pathways despite sustained inhibition of EGFR) and current strategies to overcome them (STAT3 inhibitor).

Session three was dedicated to other mutations. Federico Cappuzzo (Istituto Clinico Humanitas IRCCS, Division of Oncology, Rozzano, Italy) showed how HER2 is often deregulated in NSCLC as a consequence of overexpression, increased gene copy number or mutation. HER mutation could represent a potential relevant target in those patients as HER2 mutants NSCLC are sensitive to HER2 inhibition and combination of chemotherapy with trastuzumab. Luis-Paz Ares (Hospitales Universitarios Virgen del Rocio, Hospital General Planta Baja, Seville, Spain) gave a talk on ALK, ROS and RET gene rearrangements and the different possibilities to treat patients with such abnormalities (crizotinib, ceritinib, alectinib for ALK and ROS; cabozantinib for RET; and dacotiminib for HER1, 2 and 4) and the best place for such treatments. Nicolas Girard did the same type of presentation about target amplification/FGFR and c-MET protein overexpression with a focus on lucitanib and MET inhibitors under developments such as onartuzumab.

Session four was dedicated to a better understanding of secondary TKI resistance. Anne-Pascale Meert explained how re-biopsy, after first or subsequent therapeutic lines, is feasible and acceptable in NSCLC and useful for guiding treatment in a third of cases. In addition she discussed how the incidence of T790M-mutation at first post-TKI biopsy in EGFR-mutated NSCLC patients is up to 50\% and that patients with T790M after TKI failure could have a better prognosis than those without T790M. Christine Lovly (Vanderbilt University School of Medicine, Vanderbilt Ingram Cancer Center, Nashville, TN, USA) showed how the pre-clinical models, including cell lines and mouse models, have been helpful in informing clinical studies of patient tumour samples taken at the time of resistance. David Planchard (Dept of Medical Oncology, Gustave Roussy Cancer Campus, Roussy-Villejuif, France) reported on second and third generation inhibitors and the combination of some of these inhibitors with antibodies directed against the same target.

Session five was dedicated to new developments with presentations on how to identify the target by Céline Mascaux (Dept of Medical Oncology and Hematology, University Health Network, Princess Margaret Cancer Center, Toronto, ON, Canada), another on how to optimise the target development by Jacques Cadranel and further one on ongoing studies by Thierry Berghmans (Dept of Intensive Care Unit and Thoracic Oncology, Institut Jules Bordet, Brussels, Belgium).

Outside the discussions on each topic, two round tables allowed the audience and the faculty to have a very fruitful discussion on intergroup research development in lung-cancer targeted therapies and on research priorities. A lot of proposals have been made for next ERS researches:

1) Clinical registries for rare mutations and for toxicities of targeted therapies

2) Biobanking development for rare mutations and for rare tumours

3) Creation of a European database on prognostic factors for lung cancer

4) New research projects as tumour heterogeneity

5) Manuscript proposal for the European Respiratory Journal on "How to handle biopsies of lung cancer patients"

According to the views of the audience, the seminar was a huge success and it should be repeated in the same location in 2-years time.

\section{References}

1 Gaga M, Powell CA, Schraufnagel DE, et al. An official American Thoracic Society/European Respiratory Society statement: the role of the pulmonologist in the diagnosis and management of lung cancer. Am J Respir Crit Care Med 2013; 188: 503-507. 
2 Sculier JP, Vansteenkiste J, Schönfeld N, et al. Thoracic oncology in Europe: the ERS action plan by the Thoracic Oncology Assembly. Eur Respir J 2010; 36: 1227-1228.

3 Blum TG, Rich A, Baldwin D, et al. The European initiative for quality management in lung cancer care. Eur Respir J 2014; 43: 1254-1277.

4 Gamarra F, Boffetta P, De Ruysscher D, et al. Thoracic Oncology HERMES syllabus: setting the basis for thoracic oncology training in Europe. Eur Respir J 2013; 42: 568-571. 